\title{
A Proposal for Optomechanical Bichromatic Wavelength Switching for two-color Up-conversion Application
}

Ali Taghizad Fanid

University of Tabriz

Ali Rostami ( $\square$ rostami@tabrizu.ac.ir)

University of Tabriz https://orcid.org/0000-0002-8727-4711

\section{Research Article}

Keywords: Optomechanics, Optical force, Optical switching, Upconversion, Scattering, QDs

Posted Date: March 7th, 2022

DOI: https://doi.org/10.21203/rs.3.rs-1271526/v1

License: (c) (i) This work is licensed under a Creative Commons Attribution 4.0 International License.

Read Full License 


\title{
A Proposal for Optomechanical Bichromatic Wavelength Switching for two-color Up-conversion Application
}

\author{
A. Taghizad Fanid ${ }^{1}$ and A. Rostami ${ }^{1,2}$ \\ 1Photonics and Nanocrystals Research Lab (PNRL), University of Tabriz, Tabriz, Iran, \\ 2SP-EPT Lab., ASEPE Company, Industrial Park of Advanced Technologies, Tabriz, 5364196795, Iran.
}

\begin{abstract}
This study focuses on the Optomechanical bichromatic wavelength switching system as an indirect two-color up-conversion process that relies on optical force and nanorod scattering effects. This system is used to control light coupling between four parallel optical waveguides made of silicon nitride $\left(\mathrm{Si}_{3} \mathrm{~N}_{4}\right)$ which form two identical parts. The parallel waveguides with $0.5 \mu \mathrm{m} \times 0.5 \mu \mathrm{m}$ cross-section and $220 \mu \mathrm{m}$ lengths suspended on a silica $\left(\mathrm{SiO}_{2}\right)$ substrate embedded with the array of square silicon ( $\mathrm{Si}$ ) nanorods. By mid-IR plane wave illumination, as control light, with different intensities and different wavelengths on nanorods, scattering would increase and result in an improvement in attractive gradient optical force exerted on waveguides. Via bending waveguides toward each other, caused by optical gradient force, two different visible lights, as probe signals, propagating in the first waveguide of each section would couple to the adjacent waveguide. Simulation results reveal that when the distance between the parallel waveguides in the equilibrium position is $100 \mathrm{~nm}$ and the intensity of mid-IR light is $1.28 \mathrm{~mW} / \mu \mathrm{m}^{2}$ total coupling would occur in two situations: 1- when the control light is $4.5 \mu \mathrm{m}$, the probe light with $713 \mathrm{~nm}$ wavelength is transmitted to the output, 2 - when the control light is $3 \mu \mathrm{m}$, the probe light with $609 \mathrm{~nm}$ wavelength is transmitted to the output. In the first case $1.92 \mathrm{pN} / \mu \mathrm{m}$ optical force is needed to bend each waveguide by $9 \mathrm{~nm}$ and in the second one, $1.28 \mathrm{pN} / \mu \mathrm{m}$ optical force is needed to bend each waveguide by $6 \mathrm{~nm}$ for total coupling. The efficiency of the coupled waveguides system is \%88.6 for $609 \mathrm{~nm}$ probe light injection and \%96.5 for $713 \mathrm{~nm}$ probe light injection.
\end{abstract}

Index Terms- Optomechanics, Optical force, Optical switching, Upconversion, Scattering, QDs.

I. Introduction- Radiation pressure and gradient force are generally acknowledged to be influential upon the movement of micro and nanoparticles [1-6]. This impression is the result of light's momentum nature and its competence for transmitting the energy to matter which results in movement through interaction. Radiation pressure and gradient force as the major classification of optical force are crucial in this interaction which is closely related to momentum conservation of light. The radiation pressure force, for the direction of its motion, is parallel to light, which in turn, is because of the electromagnetic waves' bouncing back after hitting against the object. When light passes through the object, it brings about gradient force that is perpendicular to the propagation, because of refraction. The non-uniform electromagnetic field, moreover, is another reason for the origination of gradient force $[4,7,8]$.

In the $2^{\text {nd }}$ half of the $20^{\text {th }}$ century, the optical force effect on the dielectric microsphere was first observed by Arthur Ashkin in his experiments. This mechanism was continued with constructing an optical tweezer which was a method for trapping and controlling nano and microscale particles [9]. Physical, biological, and material sciences from then on used optical force effect. These fields utilized different types of optical tweezers for trapping and manipulating dielectric particles [10], metallic particles [11], biological objects such as cells, bacteria, viruses [12, 13], and nanometer precision sorting of nanoparticles [14, 15]. Plasmonic constructions have been advanced to reduce the diffraction limit of standard optical tweezers and increase gradient force in near field operations [16-18] and optomicrofluidic makeup proved to be useful for manipulating, separating, and imaging entities suspended in fluids [19-23].

Light propagation through the waveguide creates an evanescent field that may be applied in various applications. For instance, this force may be functional in trapping and manipulating biological targets by the use of nano-fibers [24] and driving nanomechanical resonators embedded in integrated silicon photonic circuits [7]. In the same vein, optical tunable micro-photonic devices employ attractive and repulsive forces arising from overlapping waves that are guided through parallel waveguides [8, 25]. Also, tuning the sign of the optical force from attractive to repulsive can be done by injecting lightwaves of different phases to coupled waveguides [26]. Using light control with light mechanisms in nano-optomechanical systems is an alternative for alloptical signal processing $[27,28]$. Still more, optical switches are further instances of light control mechanisms in which light is fundamental. All-optical fiber-chip-fiber switch operating via radiant thermal power is a case in point [29]. Optical gradient force enlarges by stirring up surface plasmon modes between graphene sheets [30] or suspended graphene over 
semiconductor/dielectric waveguide for sensing applications [31, 32]. Optical gradient force, in addition, is used to drive a twostate memory structure in a combination of a ring and a waveguide which can be used in all-optical computing [33]. The hybrid plasmonic waveguides including a combination between surface plasmon modes and waveguide modes is a similar construction. This construct is competent to enable effective optically trapping of nanometer dielectric particles [34, 35]. Another instance of gradient force among waveguides is the mid-IR to visible switching system which can be used for the indirect up-conversion process which consists of parallel waveguides suspending over a substrate and embedded with metal-dielectric nanoparticles [36]. Wavelength up-conversion is typically a nonlinear optical event in which energy transfer happens. Such an enhancement in wavelength is done by irradiating low energy waves to nonlinear materials and by emitting higher energy waves [37, 38].

The exact measurement of movement on a nanometer scale needs big scrutiny. An instance of this is mechanical motion in the fiber-taper NOM system which causes the Femto-newton sensing of the optical force on nanofiber [39, 40].

Different scattering behaviors are seen in the interaction of electromagnetic waves with nano-objects at wavelength ranges from visible to infrared such as the scattering profiles of a silicon nanosphere with a radius of $75 \mathrm{~nm}$ changing with light illumination in the visible spectral range [41]. Also, exciting single core-shell nanoparticles with various sizes cause interference between different orders of the electric and magnetic moment. This interaction takes place in specified circumstances i.e. $a_{n}=b_{n}$ (Mie scattering coefficients) and could lead to elimination scattering in the backward direction and improving scattering in the forward orientation. In addition, more advancement appears in forward direction by higher-order interferences of magnetic and electric modes [42]. Further, for the amount of scattering in the forward direction to reach zero, we may use gain-assisted dielectric shell-coated metallic core spherical particles [43].

This paper aims to design and simulate a bichromatic optomechanical system to convert two mid-IR wavelengths to two short wavelengths in the visible range. This system is used to control light coupling between four parallel optical waveguides which form two identical parts. The conversion operation is done by switching method and there is no energy transfer between the mid-IR input wavelengths and the visible lights propagating through the waveguides, unlike the usual frequency up-converters in which the energy transfer takes place. In this optomechanical system, nanorods have been used to increase the optical force exerted on couped waveguides. Mid-IR illumination over nanoparticles and the contribution of scattering effect would increase the optical force applied on the waveguides.

II. Structure- Fig. 1 schematically illustrates the basic structure of the nano Optomechanical bichromatic up-conversion system. This structure consists of two identical parts which are used to control light coupling between four parallel optical waveguides. In each section two doubly clamped parallel waveguides made of $\mathrm{Si}_{3} \mathrm{~N}_{4}$ with $\mathrm{L}=220 \mu \mathrm{m}$ length and $0.5 \mu \mathrm{m} \times 0.5 \mu \mathrm{m}$ cross-section are suspended $\mathrm{h}=1025 \mathrm{~nm}$ above the nanorods. The gap between the parallel waveguides in the equilibrium position is $100 \mathrm{~nm}$. The Si square nanorods with the size of $s=1050 \mathrm{~nm}$ and triplet arraying method are placed on a glass substrate as a transparent plate to a high-power mid-IR light pump. They are arranged at a certain distance from each other i.e. $a=0.5 \times s=525 \mathrm{~nm}$ in the order of the size of nanorods. The intensity of the mid-IR pump as control light is about $1.28 \mathrm{~mW} / \mu \mathrm{m}^{2}$ and its wavelengths are $4.5 \mu \mathrm{m}$ and $3 \mu \mathrm{m}$. Visible lights with $713 \mathrm{~nm}$ and 609 wavelengths as probe signals with arbitrary power are injected into the entrance of parallel waveguides in two sections. The distance between the two sections $(p=15 \mu \mathrm{m})$ is chosen so that the scattering caused by the nanorods does not affect the waveguides of the other section.

The emission of intermediate infrared light waves with wavelengths of $3 \mu \mathrm{m}$ and $4.5 \mu \mathrm{m}$, creates different attractive forces between the paired waveguide and the electric field magnitude between them would increase, considering the backward scattering from the nanorods. As a result, raising the applied optical force would cause the waveguides to deviate. With bending both adjacent waveguides towards each other about $9 \mathrm{~nm}$, visible light with $713 \mathrm{~nm}$ wavelength that is passing through the first waveguide would couple to another and may be observed in the output of the neighboring waveguide. besides, if the deflection is $6 \mathrm{~nm}$, the other probe signal with $609 \mathrm{~nm}$ wavelength can be transmitted to the output.

For using scattering nanorods in certain mid-IR wavelengths, i.e. $3 \mu \mathrm{m}$ and $4.5 \mu \mathrm{m}$, various square rodes were simulated. In the process of this simulation, changing the side size of the square silicon rod indicated that when the size is 1050nm, scattering in the mid-IR range raises. Other parameters such as the gap between waveguides (d), the height of the waveguides above the surface of the Si nanorod array (h), are modified through several simulations. It needs to mention that the distance between the nanorods (a) is approximately the same as the side size of nanorods. 

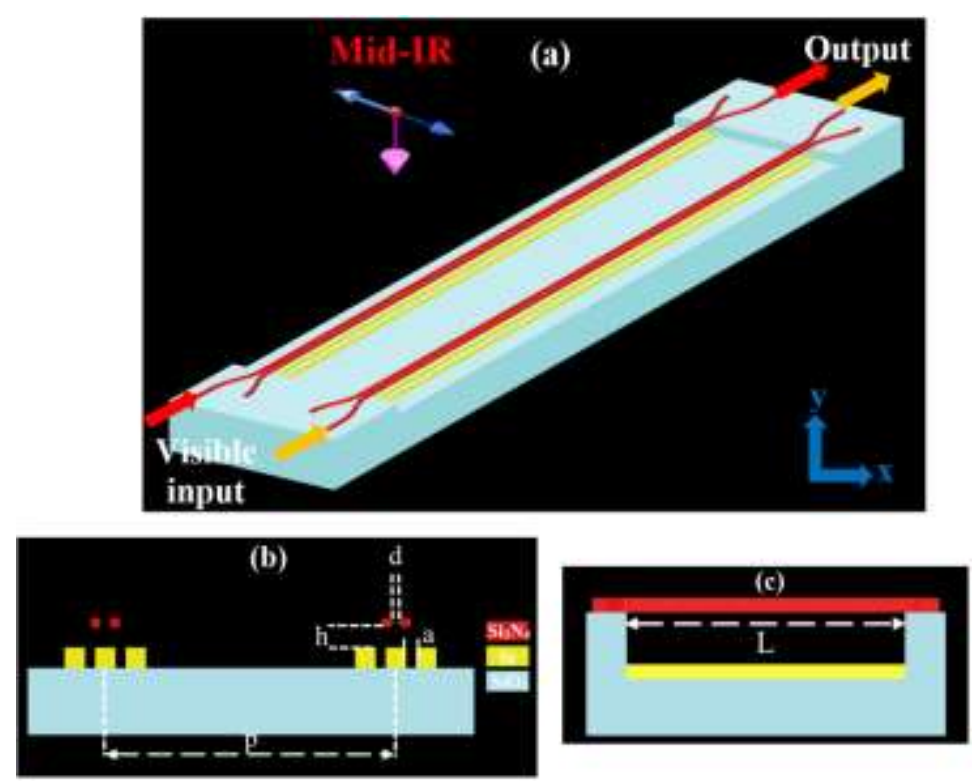

Fig. 1. a) 3D Schematic of nano Optomechanical bichromatic up-conversion system. Triplet Array of Silicon nanorods with the side size of $1050 \mathrm{~nm}$ is placed on a $\mathrm{SiO}_{2}$ substrate. Four $\mathrm{Si}_{3} \mathrm{~N}_{4}$ waveguides with dimensions $220 \mu \mathrm{m} \times 0.5 \mu \mathrm{m} \times 0.5 \mu \mathrm{m}$ are suspended above the nanorods and consist of two identical parts with two coupled waveguides. The intensity of the mid-IR Pump is about $1.28 \mathrm{~mW} / \mu \mathrm{m}^{2}$ and its wavelengths are $4.5 \mu \mathrm{m}$ and $3 \mu \mathrm{m}$. Visible lights with 713 (red) nm and 609 (orange) wavelengths as probe signals are injected into the entrance of parallel waveguides in two sections. b) $x$-y view of the structure, $d$ is the distance between parallel-coupled waveguides, $h$ is waveguides' height from the top of the nanorods, a is the distance between nanorods, $p$ is the distance between two sections of the structure. $c$ ) $z-y$ view of the structure, $L$ is the length of suspended waveguides.

III. Methodology- For the investigation of the optomechanical two-color up-conversion mechanism, we studied two physical theories i.e. optical force and scattering by nanorods.

\section{A. Optical force}

In this research, the optical force exerted on the coupled waveguides has been used as a driver for deflecting of waveguides, therefore, it should be calculated on the desired volume. Here, the optical force is calculated by FDTD (finite difference time domain) numerical solution method by integrating the Maxwell stress tensor over the cross-section of each waveguide separately in the $x-y$ plane. Maxwell's stress tensor is described as follows [32]:

$\psi_{i, j}=\varepsilon_{0}\left(E_{i} E_{j}-\frac{1}{2} \delta_{i j} E^{2}\right)+\frac{1}{\mu_{0}}\left(B_{i} B_{j}-\frac{1}{2} \delta_{i j} B^{2}\right)$

In Eq. (1), $i, j \in x, y, z, E_{i}$, and $B_{i}$ are the amplitude of electric field intensity and magnetic flux density, $\delta_{i, j}$ is Kronecker delta function, and $\psi_{i, j}$ is MST element at a single point.

\section{B. Scattering of nanoparticles}

Irradiated light waves interact with the nanorods placed on the $\mathrm{SiO}_{2}$ substrate and scatter the light in the backward and forward direction which increases the strength of the electric field between the coupled waveguides. As the micro and nanoscale particles move towards a stronger field intensity [16], the attractive force due to the scattering of nanorods will appear as well. Theoretically, Mie theory is used to analyze the scattering spherical particles which have been influenced by electromagnetic waves. Scattering efficiency (scattering cross-section divided by the cross-section of the particle) for spherical and core-shell objects is [42-44]:

$$
Q_{s c a}=\frac{2}{k^{2} R^{2}} \sum_{n=1}^{\infty}(2 n+1)\left(\left|a_{n}^{2}\right|+\left|b_{n}{ }^{2}\right|\right)
$$

In Eq. (2), k, R, an, and $b_{n}$ are the wavenumber, the radii of the most ou layer of the core-shell object, Mie scattering coefficients which refer to $\mathrm{n}^{\text {th }}$ electric and magnetic moments respectively. Also, scattering intensity (SI) in the far-field region is as follows [42-44]:

$$
S I(\theta, \varphi)=\frac{\lambda^{2}}{4 \pi^{2} d^{2}}\left[\left|T_{1}(\cos \theta)\right|^{2} \sin ^{2} \varphi+\left|T_{2}(\cos \theta)\right|^{2} \cos ^{2} \varphi\right]
$$

In Eq. (3), $\lambda$ is the incident wavelength, $d$ is the distance from to the position where the intensity of scattering is measured to the center of the spherical particle, $\theta$ is polar angel and $\varphi$ is the azimuthal angle. $T_{1,2}(\cos \theta)$ are detailed to: 
$T_{l}(\cos \theta)=\sum_{n=1}^{\infty} \frac{2 n+1}{n(n+1)}\left[a_{n} \pi_{n}(\cos \theta)+b_{n} \tau_{n}(\cos \theta)\right]$

$T_{2}(\cos \theta)=\sum_{n=1}^{\infty} \frac{2 n+1}{n(n+1)}\left[a_{n} \tau_{n}(\cos \theta)+b_{n} \pi_{n}(\cos \theta)\right]$

which $\pi_{n}(\cos \theta)=P_{n}^{l}(\cos \theta) / \sin \theta, \tau_{n}(\cos \theta)=d P_{n}^{l}(\cos \theta) / d \theta$ and $P_{n}^{l}(\cos \theta)$ is the first order of Legendre function.

In this work, the scattering efficiency of nanorods is calculated with the FDTD solution method in the cross-section of nanorods in the $x-y$ plane.

IV. Results and Discussion- To investigate and simulate the structure which is used in the article, several procedures have been employed. The optical force over coupled waveguides and the scattering efficiency of a group of Si nanorods are calculated by the numerical FDTD solution process. The modal analysis and coupling between adjacent waveguides are computed through FDE (finite difference Eigenmo) method. Additionally, the finite element method (FEM) is utilized to specify the mechanical properties of $\mathrm{Si}_{3} \mathrm{~N}_{4}$ nanowire.

\section{A. Scattering of nanorods}

FDTD simulation results are obtained for the array of Si nanorods that are lying upon the silica substrate in fig. 2. By mid-IR frequency range illumination of the nanorods, as fig. 2a indicates, the amount of scattering in the forward and backward direction varies concerning the different input wavelengths. In some wavelengths, i.e. $3 \mu \mathrm{m}$ and $4.5 \mu \mathrm{m}$, the scattering rate is increased in the backward direction which can be used in the structure of fig. 1 to increase the electric field strength between coupled waveguides (fig. 2b). Arranging the nanorods causes the field to be more oriented in the forward or backward directions. For example, Fig. $2 \mathrm{~d}$ and $2 \mathrm{e}$ show the distribution of the total electric field magnitude $(\mathrm{V} / \mathrm{m})$ (including the input field and the scattered field) for a single nanorod and a triple array of nanorods, respectively. In the nanowire array mode, the field amplitude above the nanorods is increased compared to the single-mode. The data of the material properties are set according to reference [45] for $\mathrm{Si}$ and $\mathrm{SiO}_{2}$ and reference [46] for $\mathrm{Si}_{3} \mathrm{~N}_{4}$. The minimum size of the mesh is set to $10 \mathrm{~nm}$ and all of the boundary conditions are set as PML (perfect matched layer).

(a)

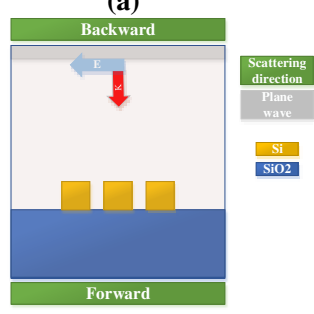

(b)

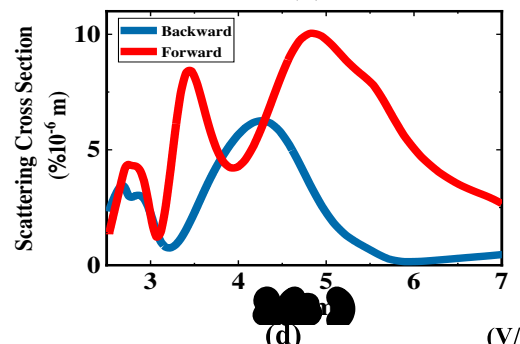

(d)

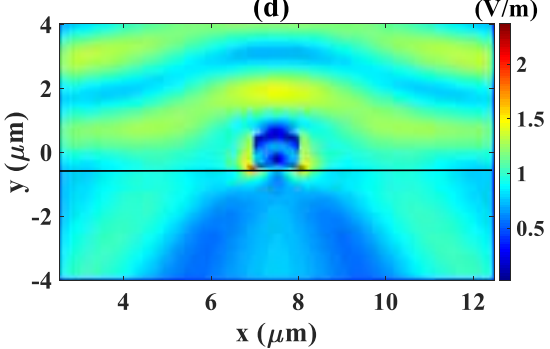

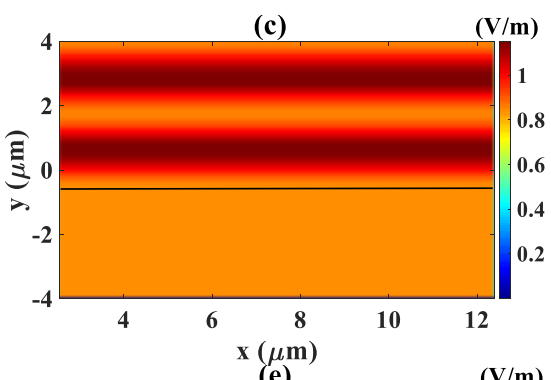

(e)

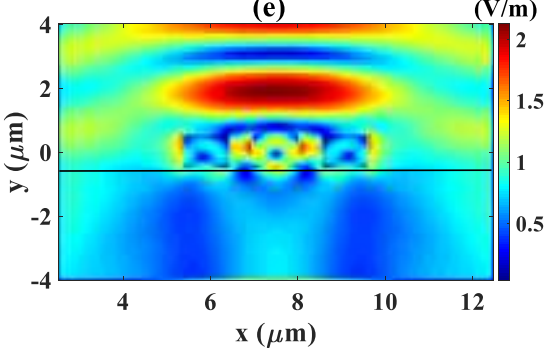

Fig. 2. FDTD Simulation results of single and an array of Si nanorods with side size of $s=1050 \mathrm{~nm}$ in the $x-y$ plane. The distance between nanorods is a $=$ $525 \mathrm{~nm}$. a) Schematic of an incident plane wave on an array of Si nanorods in which electric field is polarized in the $x$-direction and propagate in the $y$-direction (the amplitude of electric field is $1 \mathrm{~V} / \mathrm{m}$ ). b) Calculating scattering cross-section. c) Total electric field magnitude distribution at $4.5 \mu \mathrm{m}$ without considering nanorods on the substrate. $d$, e) Total electric field magnitude distribution (mostly backward direction) at $4.5 \mu \mathrm{m}$ wavelength. 


\section{B. Optical force}

In the proposed bichromatic optomechanical system, when the mid-IR plane wave is illuminated from the top of the structure on the nanorods (fig. 1a) and light interacts with them, it causes the nanorods to scatter and increase the attractive optical force among the suspended coupled waveguides. Surveying the simulation results shows that the force exerted on the coupled waveguides depends on the input mid-IR wavelength and the vertical distance between waveguides and the top of the nanorods. Indeed, the various gap between coupled waveguides and the side size of the nanorods would change the applied optical force. The total force exerted on the coupled waveguides is obtained by integrating the MST over each waveguide.

The results obtained in Fig. 3 show that the electromagnetic wave radiation on the structure of fig. 1 increases the electric field strength between the coupled waveguides. For example, in fig. $3 \mathrm{~b}$, fig $3 \mathrm{c}$, and fig. $3 \mathrm{~d}$ a comparison is made for the case where the input mid-IR wavelength is $3 \mu \mathrm{m}$ and $4.5 \mu \mathrm{m}$. It is worth mentioning that where the amount of scattering is high, i.e. $4.5 \mu \mathrm{m}$, the field intensity is also higher between the coupled waveguides.

Fig. 4 explains the simulation outcome dealing with the optical force in the optomechanical up-conversion system that is shown in Fig. 1a. The structure is made up of a triplet arrangement of nanorods to cover the whole portion of the paired waveguides. Moreover, the $220 \mu \mathrm{m}$ span of the square nanorods in the z-direction provides the condition for applying uniform optical force along the close waveguides. Also, the 3-bits in the x-direction ensures the symmetry of the exerted optical force. The data of the optical properties are determined according to reference [45] for $\mathrm{Si}$ and $\mathrm{SiO}_{2}$ and reference [46] for $\mathrm{Si}_{3} \mathrm{~N}_{4}$. The minimum dimensions of the mesh unit are $10 \mathrm{~nm}$ and entire of the boundary conditions are PML.

Fig. 4a shows making changes to the Mid-IR input wavelength or the gap between coupled waveguides alters the amount of applied optical force. For example, when the entering pump intensity is I = $1 \mathrm{~mW} / \mu \mathrm{m}^{2}$, different gaps ranging from $\mathrm{d}=100 \mathrm{~nm}$ to $d=300 \mathrm{~nm}$, would cause the waveguides to experience different forces. Indeed by reducing the distance of the adjacent waveguides in some wavelengths, the applied force increases. Since the waveguides' approach to one another is noteworthy, optical force is computed in the $x$-direction. Also by the symmetry of the optomechanical-system construction, the optical force applied on the coupled guides is identical in amplitude but reverse in direction.

To probe the action of scattering effect on the exerted optical force, the structure (fig. 1a) is emitted with mid-IR wavelengths by eliminating the nanorods. A comparison between the issues, fig. $4 \mathrm{a}$ and fig. $4 \mathrm{~b}$, shows that the force will be multiplied in several frequencies.

Changing the dimension of $\mathrm{Si}_{3} \mathrm{~N}_{4}$ nanofibers affects the force applied to the coupled waveguides. Fig. $4 \mathrm{c}$ shows that increasing the size of nanofibers causes the wavelength at which the input force is maximum to shift slightly toward larger wavelengths. Also changing the distance of the coupled waveguides from the top surface of the nanorods results in changing the applied optical force, which is because of the type of electric field distribution above the nanorods (fig. $4 \mathrm{~d}$ ). By examining the results obtained in fig. 4, the values of parameters are determined; $d=100 \mathrm{~nm}, \mathrm{~s}=1050 \mathrm{~nm}, \mathrm{~h}=1025 \mathrm{~nm}$. The value of $d$ is considered to be 100 $\mathrm{nm}$ for applying the maximum amount of optical force. The value of $\mathrm{s}$ is set to $1050 \mathrm{~nm}$ so that the force applied at the wavelength of $4.5 \mu \mathrm{m}$ has its maximum value. We also change the height to reach the desired amount of force more precisely. Fig. 5, also, shows the results by considering the parameters as determined values.
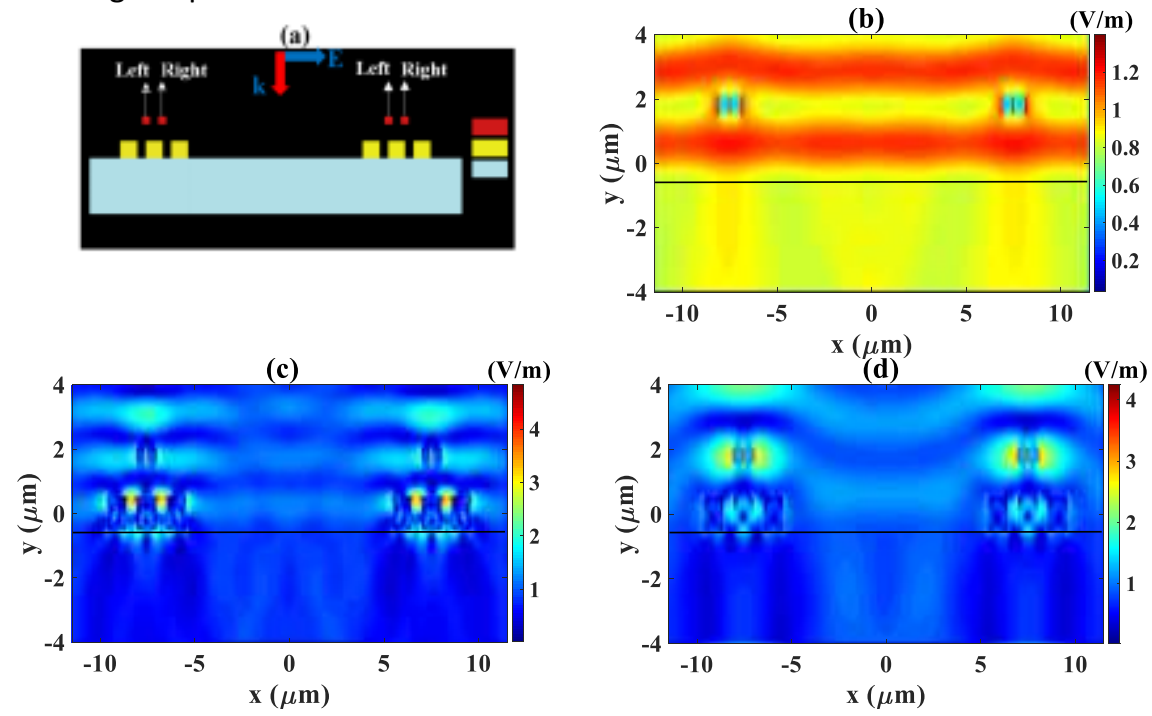

Fig. 3. Calculating of the electric field magnitude in the surface of the cross-section of the structure (shown in fig. 1a). a) Schematic of an incident plane wave on an array of Si rods in which electric field polarized in the $x$-direction and propagate in the $y$-direction (the amplitude of electric field is $1 \mathrm{~V} / \mathrm{m}$ ). b) Here nanorods are removed and the input mid-IR wavelength is $\lambda=4.5 \mu \mathrm{m}$. c) The input mid-IR wavelength is $\lambda=3 \mu \mathrm{m}$ and d) the input mid-IR wavelength is $\lambda=4.5 \mu \mathrm{m}$. 

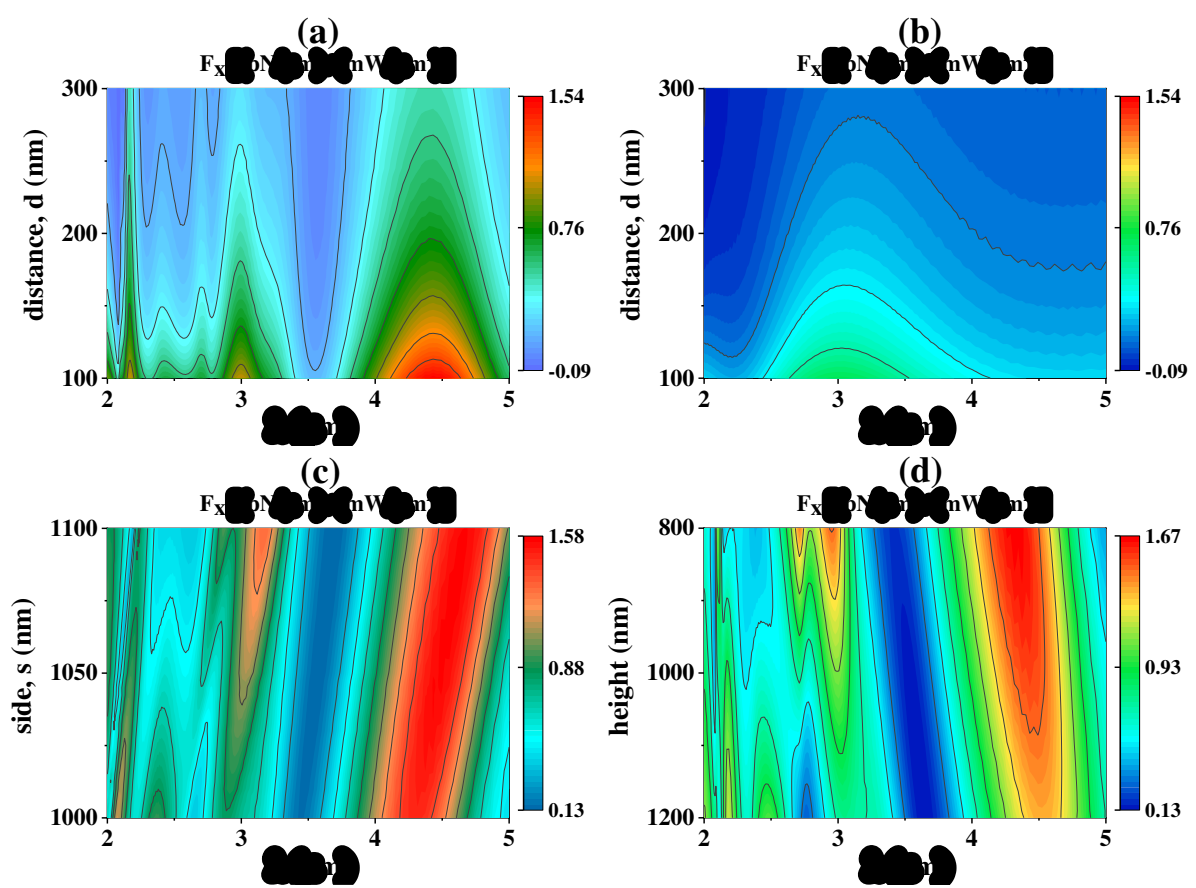

Fig. 4. FDTD Simulation results of nano Optomechanical bichromatic up-conversion system is shown in fig. 1; Calculating the total force in the x-direction exerted on the left waveguides (shown in fig. 3a) by integrating the MST over the cross-section of the waveguide. The incoming light intensity is $1 \mathrm{~mW} / \mu \mathrm{m}^{2}$ with different wavelengths (horizontal axes). a) Changing the distance of the coupled waveguides from each other which is specified by the parameter $\mathrm{d}$. b) Changing the distance of the coupled waveguides from each other without considering the nanorods. In these cases, the size of the nanorods is $\mathrm{s}=1050 \mathrm{~nm}$ and the height is $h=1025 \mathrm{~nm}$. c) Changing the size of nanorods which is specified by parameter s and assuming $d=100 \mathrm{~nm}$ and $\mathrm{h}=1025 \mathrm{~nm}$. d) Changing the distance of the waveguides from the surface of the nanorods and assuming $d=100 \mathrm{~nm}$ and $\mathrm{a}=525 \mathrm{~nm}$.

Fig. 5. FDTD Simulation results of nano Optomechanical bichromatic up-conversion system is shown in fig. 1; Calculating the total force in the $x$-direction exerted on both coupled waveguides (shown fig. 3a) by integrating the MST over the cross-section of the waveguide. The incoming light intensity is $1 \mathrm{~mW} / \mu \mathrm{m}^{2}$ with different wavelengths (horizontal axes). Comparing the structure in the cases that nanorods are present and not present.

\section{Mechanical properties of the waveguides}

This section investigates the simulation results of mechanical behavior of single $\mathrm{Si}_{3} \mathrm{~N}_{4}$ with dimensions of $220 \mu \mathrm{m} \times 0.5 \mu \mathrm{m} \times 0.5 \mu \mathrm{m}$, using the FEM solution method. Poisson's ratio is 0.23 for $\mathrm{Si}_{3} \mathrm{~N}_{4}$, Young's module is $250 \mathrm{GPa}$, and density is $3100 \mathrm{~kg} / \mathrm{m} 3$ [47]. As the waveguide is exerted with different forces, the stationary response of the amount of deflection on the line crossing the middle of the waveguide and at its center can be calculated. Because of the small deformation of the waveguide compared to its dimensions, displacement may be assumed linear (fig. $6 \mathrm{~b}$ and fig. 6c). Besides fig. $6 \mathrm{~b}$ provides the optical force that is required for the displacement desired. 


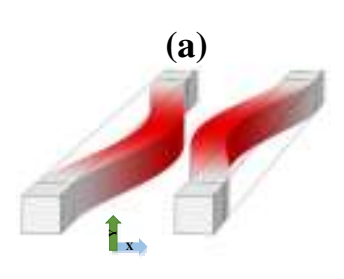

(b)

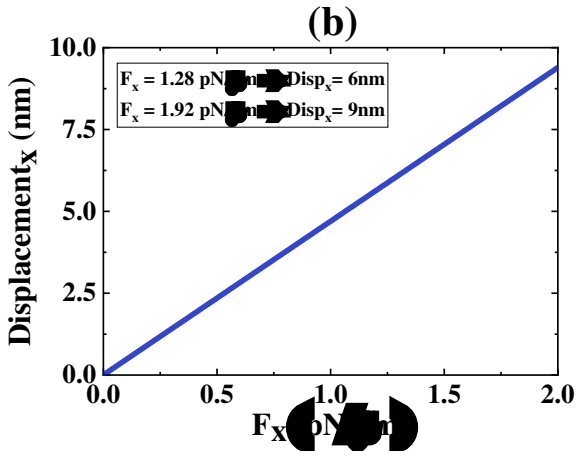

(c)

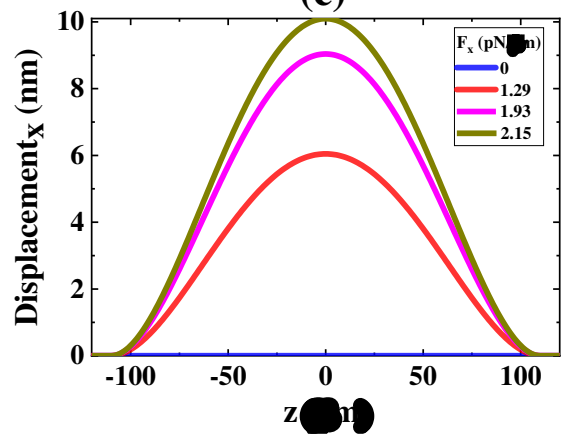

Fig. 6. FEM simulation results of single $\mathrm{Si}_{3} \mathrm{~N}_{4}$ waveguide with dimensions of $220 \mu \mathrm{m} \times 0.5 \mu \mathrm{m} \times 0.5 \mu \mathrm{m}$, the exerted force is in the $\mathrm{x}$-direction; a) Schematic of the

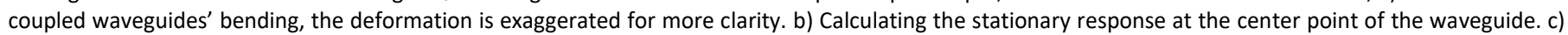
Calculating the stationary response on the line crossing the middle of the waveguide.

\section{Coupling between waveguides}

To investigate how light is coupled between suspended waveguides, light with two different wavelengths in the visible range is injected into the first waveguide of each section (fig. 7a). By changing the gap between the coupled waveguides, the amount of light power propagating and coupling will change from the first waveguide to the second one (fig. $7 \mathrm{~b}-7 \mathrm{~g})$. In fig. 7 , either of the right and left columns belongs to a section of the structure. In fig. $7 \mathrm{~b}$, fig. $7 \mathrm{~d}$, and fig. $7 \mathrm{f}$, the visible light with $713 \mathrm{~nm}$ wavelength is injected to the first (upper) waveguide; results reveal that in case the distance of the waveguides is equal to $82 \mathrm{~nm}$, the coupling occurs and the signal is transmitted into the end of the second waveguide, which is considered as the output of section 1 . But in the other two cases, i.e. $d=100 \mathrm{~nm}$ and $d=88 \mathrm{~nm}$, the optical power is not transmitted to the output. Meanwhile, in fig. 7c, fig. $7 \mathrm{e}$, and fig. $7 \mathrm{~g}$, the visible light with $713 \mathrm{~nm}$ wavelength is injected into the first waveguide. In this case, the power of light is transmitted to the output just when the distance is $\mathrm{d}=88 \mathrm{~nm}$ and in the other two situations, the output is low.

It is worth mentioning that to obtain the two visible wavelengths $\lambda=713 \mathrm{~nm}$ and $\lambda=609 \mathrm{~nm}$, as well as the locations, $\mathrm{d}=82 \mathrm{~nm}$, $\mathrm{d}=88 \mathrm{~nm}$, and $\mathrm{d}=100 \mathrm{~nm}$, where the waveguides will reach equilibrium after applying force, the FDE solution method is functional. Through these simulations, the transmittance vs. wavelength and distance is shown in fig. 8.

Finally, based on the diagrams shown in fig. 4, fig. 6b, and fig. 7, we conclude that a graph can be derived showing the transmittance of the probe lights compared to the mid-IR light input intensity (fig. 9). In this way, first, the waveguides for both sections are at the state of equilibrium at a distance of $100 \mathrm{~nm}$ from each other and then the mid-IR plane waves would create an optical force, causing coupled waveguides to attract one another. If the mid-IR light intensity is considered to be 1.28 $\mathrm{mW} / \mu \mathrm{m}^{2}$, the radiation of two different wavelengths $(4.5 \mu \mathrm{m}$ or $3 \mu \mathrm{m})$ will result in the detection of two distinct visible lights $(713 \mathrm{~nm}$ or $609 \mathrm{~nm}$ ) in the output. The efficiency of the coupled waveguides system is \%88.6 for $609 \mathrm{~nm}$ probe light injection and $\% 96.5$ for $713 \mathrm{~nm}$. Table.1, also, indicates significant results obtained from the system. The minimum dimension of the mesh unit between the coupled waveguides is set to $1 \mathrm{~nm}$ and $5 \mathrm{~nm}$ for other regions. The entire boundary conditions are PML.

According to the simulations, the probe light is introduced from the entrance of the excited waveguide. This can be accomplished with gratings $\mathrm{Si}_{3} \mathrm{~N}_{4}$ upon the $\mathrm{SiO}_{2}$ substrate as grating couplers that are used to couple the probe signal into the waveguide [48, 49]. In addition, the layer fabrication process can rely on planner technology, such as lithography and etching [50].
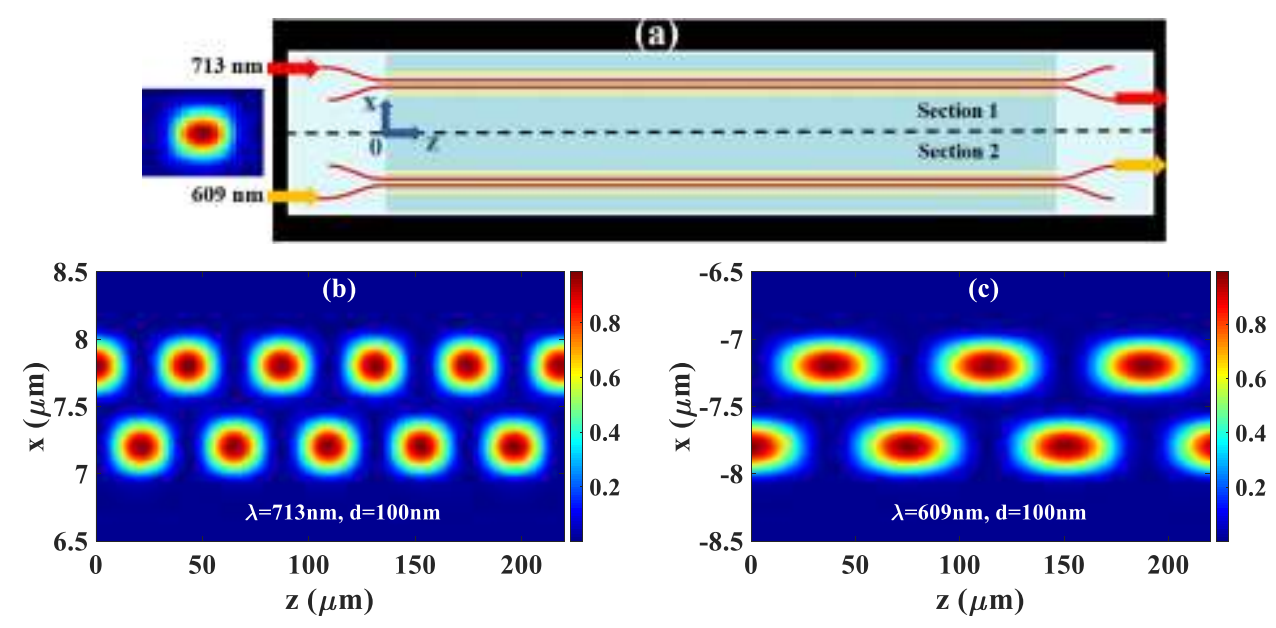

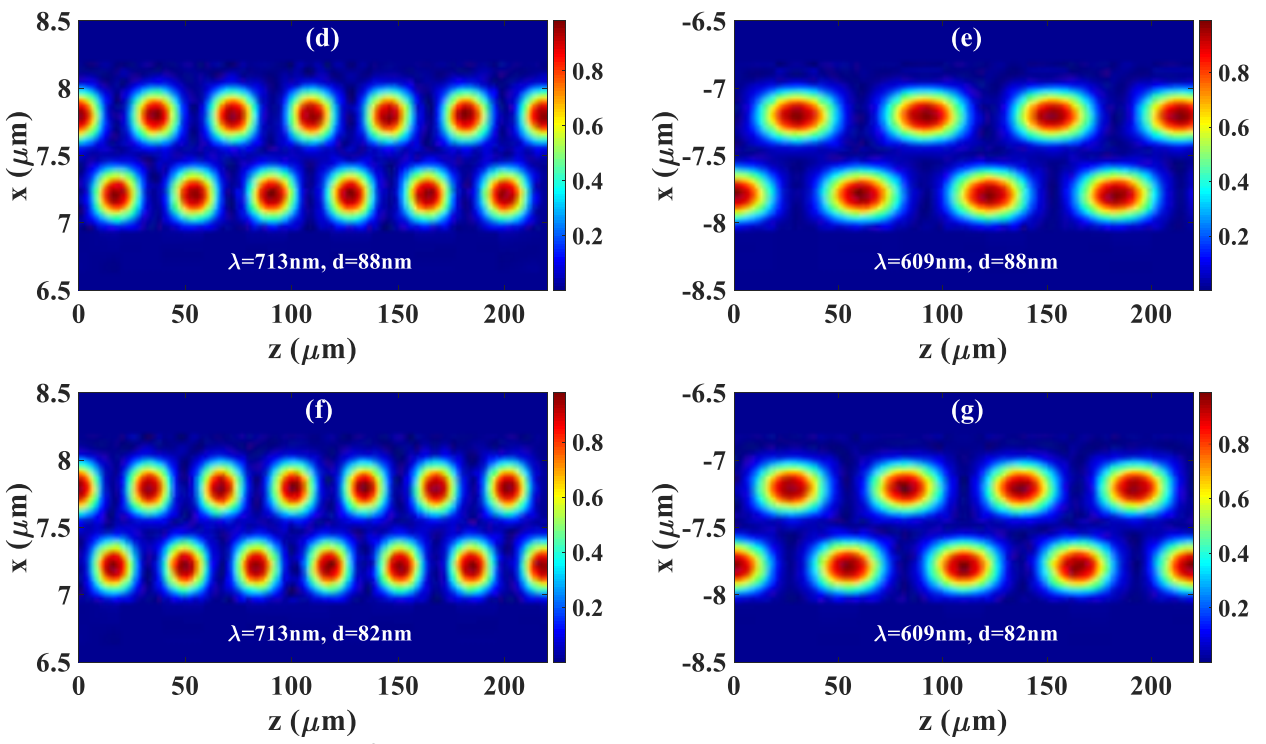

Fig. 7. 3D, FDE simulation results; calculating the electric field magnitude propagating among the excitation waveguide and the coupling waveguide on the $z-x$ plane crossing through the total length of the coupled waveguides. a) TE mode profile is injected into the first waveguide in the z-direction with $713 \mathrm{~nm}$ wavelength for section 1 and $609 \mathrm{~nm}$ wavelength for section 2 . The electric field amplitude is $1 \mathrm{~V} / \mathrm{m}$ and polarized the $y$-direction. $b$, c) The distance between the waveguides is $d=100 \mathrm{~nm}$. $d$, e) The distance between the waveguides is $d=88 \mathrm{~nm} . f, g$ ) The distance between the waveguides is $d=82 \mathrm{~nm}$

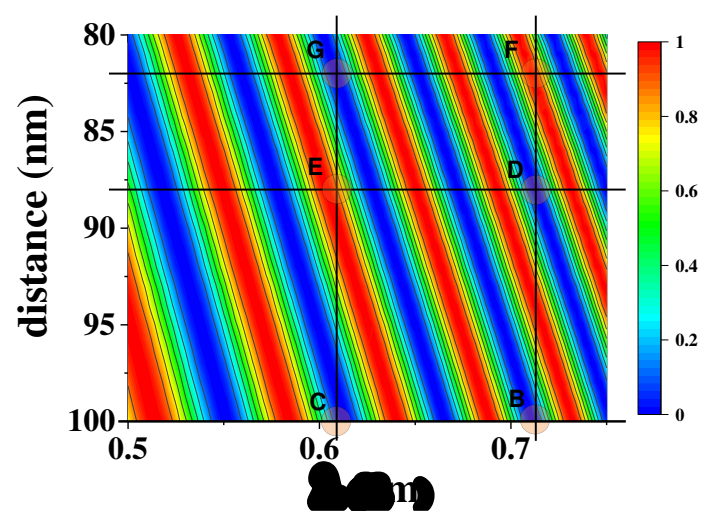

Fig. 8. The Simulation results in with FDE method to calculate the output power of visible lights detected in two parts at the end of the second waveguide. 4 points, according to the intersecting lines, indicate the wavelengths and distances for use in switching two wavelengths, which are: B, C, D, E, F, G. the injected visible light is TE mode and the electric field is polarized in the $y$-direction.
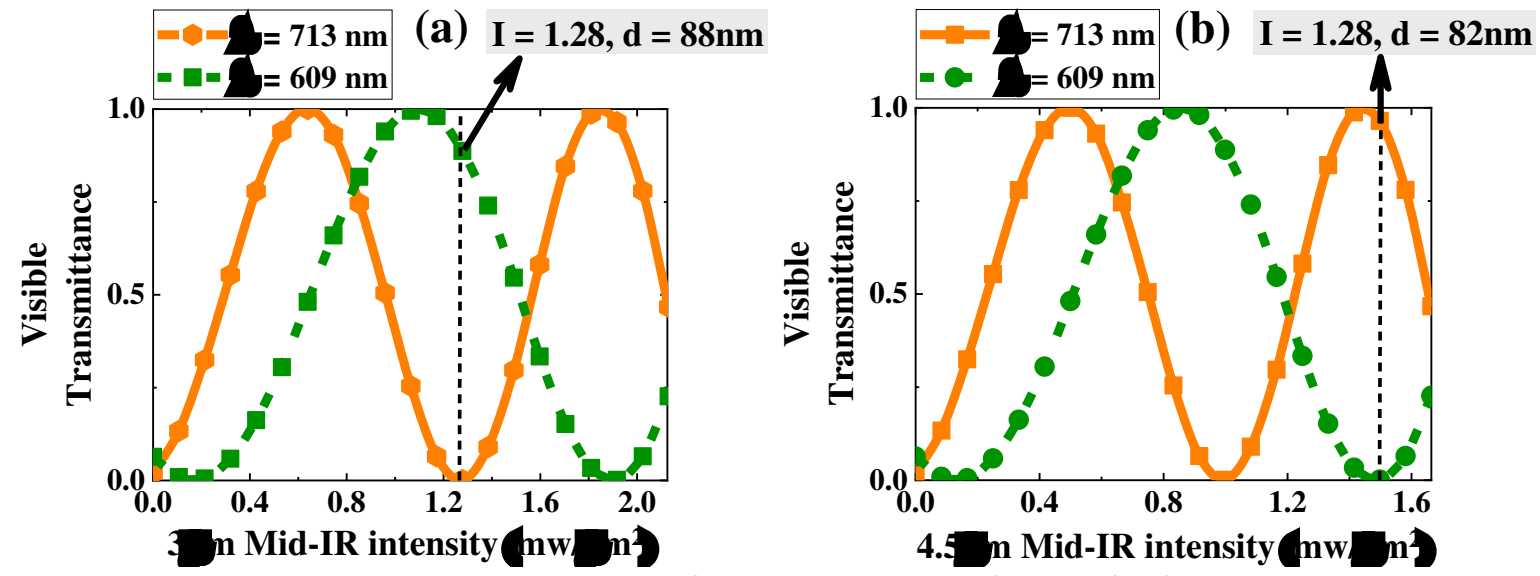

Fig. 9. FDTD Simulation results; the power passing through the output of the coupling waveguides (shown in fig. 7) vs. Mid-Ir intensity. The orange line (refer to $\lambda=713 \mathrm{~nm}$ ) indicates the output of section 1 and the green line (refer to $\lambda=609 \mathrm{~nm}$ ) indicates the output of section 2 . The equilibrium position of the coupled waveguides is $d=100 \mathrm{~nm}$ when mid-IR power is equal to 0 . Assuming the intensity equal to $1.28 \mathrm{~mW} / \mu \mathrm{m}_{2}$ the deflection of each waveguide is $9 \mathrm{~nm}$ and $6 \mathrm{~nm}$ when mid-IR wavelengths are $4.5 \mu \mathrm{m}$ and $3 \mu \mathrm{m}$ respectively. 


\begin{tabular}{|c|c|c|c|c|c|}
\hline 1 & 2 & 3 & 4 & 5 & 6 \\
\hline Wavelength & Non. & mid $-I R=4.5 \mu \mathrm{m}$ & mid-IR=3.5 $\mu \mathrm{m}$ & $\mathrm{N}: \mathrm{Si}_{3} \mathrm{~N}_{4}$ & $\operatorname{Loss}(\mathrm{dB} / \mathrm{cm}): \mathrm{Si}_{3} \mathrm{~N}_{4}$ \\
\hline $713 \mathrm{~nm}$ & Low (\%2) & High (\%96.5) & Low (\%0.1) & 2.0307 & 0.00043 \\
\hline $609 \mathrm{~nm}$ & Low $(\% 6.4)$ & Low $(\% 0.1)$ & High (\%88.6) & 2.0419 & 0.00065 \\
\hline
\end{tabular}

Table. 1. The results obtained for the system are shown in fig. 1 . Column 1 lists the wavelengths injected into the waveguides. Columns 2 to 4 show the system outputs for the different wavelengths of the input Mid-IR pump, the intensity is $1.28 \mathrm{~mW} / \mu \mathrm{m}^{2}$. Column 5 is the refractive index of the Siz $\mathrm{N}_{4}$ at two wavelengths on which the simulations are done. Columns 6 is the loss of coupling waveguides with $d=100 \mathrm{~nm}$ and a single $\mathrm{Si}_{3} \mathrm{~N}_{4}$ guide on the substrate. The input intensity is $1.28 \mathrm{~mW} / \mathrm{\mu m}^{2}$.

Conclusion- This study surveys the effect of the scattering of electromagnetic fields by the array of square nanorods on an opticalforce-based optomechanical system. This system can be used for the bichromatic mid-IR to visible up-conversion process. The study uses two different mid-IR wavelengths as control and two distinct visible waves as probe signals as well. Simulation results illustrate that scattering of nanorods would increase the optical force between suspended coupled waveguides for two identical sections of the structure. When the sufficient mid-IR pump light $\left(I=12.8 \mathrm{~mW} / \mu \mathrm{m}^{2}\right)$ is provided, by selecting the wavelengths between $4.5 \mu \mathrm{m}$ or $3 \mu \mathrm{m}$, the detected light in the output would be $\lambda=713 \mathrm{~nm}$ and $\mathrm{nm} \lambda=609 \mathrm{~nm}$, respectively. According to the findings of this research, the scattering of nanoparticles enhances the applied optical force in the optomechanical system. The proposed system can be used as a two-color up-conversion system at room temperature.

\section{References}

[1] M. Metcalfe, "Applications of cavity optomechanics," Applied Physics Reviews, vol. 1, no. 3, p. $031105,2014$.

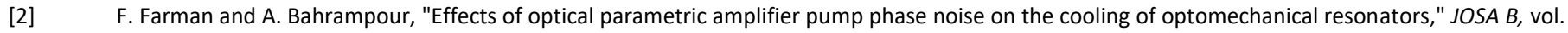
30, no. 7, pp. 1898-1904, 2013.

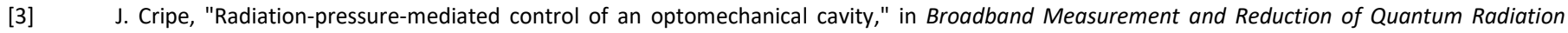
Pressure Noise in the Audio Band: Springer, 2020, pp. 67-79.

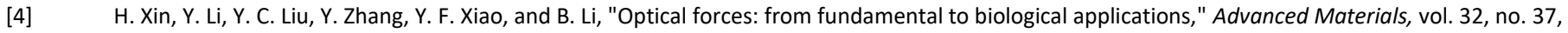
p. 2001994, 2020.

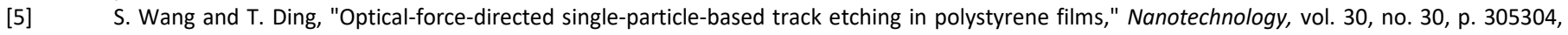
2019.

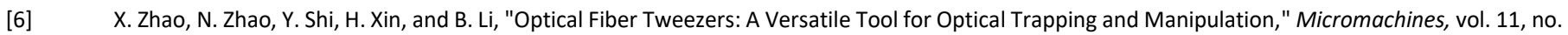
2, p. 114, 2020.

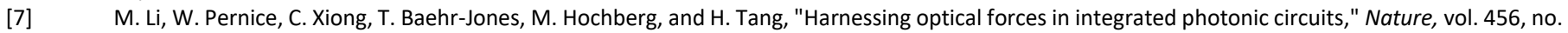
7221, p. 480, 2008.

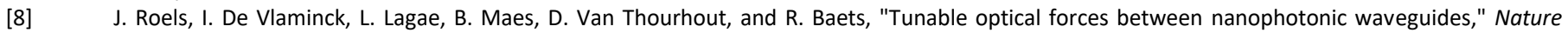
nanotechnology, vol. 4, no. 8, p. 510, 2009.

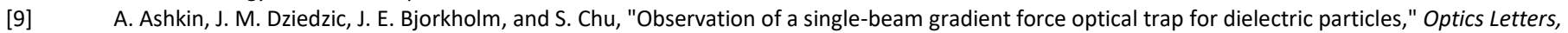
vol. 11, no. 5, pp. 288-290, 1986.

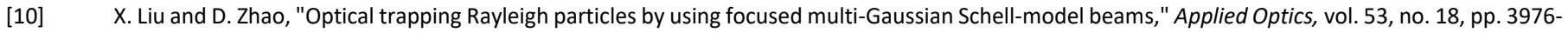
3981, 2014.

[11] F. Hajizadeh and S. N. S. Reihani, "Optimized optical trapping of gold nanoparticles," Optics Express, vol. 18, no. 2, pp. 551-559, 2010.

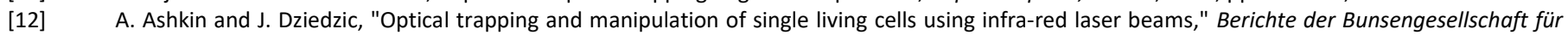
physikalische Chemie, vol. 93, no. 3, pp. 254-260, 1989.

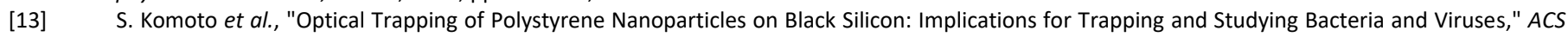
Applied Nano Materials, vol. 3, no. 10, pp. 9831-9841, 2020.

[14] Y. Shi et al., "Nanometer-precision linear sorting with synchronized optofluidic dual barriers," Science advances, vol. 4, no. 1, p. eaao0773, 2018.

[15] F. Nan and Z. Yan, "Sorting metal nanoparticles with dynamic and tunable optical driven forces," Nano Letters, vol. 18 , no. 7, pp. 4500-4505, 2018.

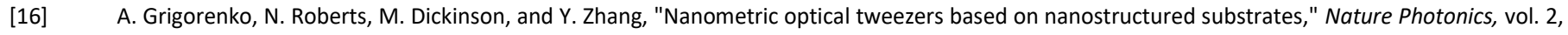
no. 6, p. 365, 2008.

[17] M. L. Juan, M. Righini, and R. Quidant, "Plasmon nano-optical tweezers," Nature photonics, vol. 5, no. 6, p. 349, 2011.

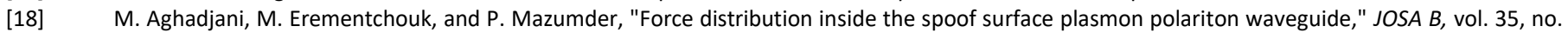
5, pp. 1113-1118, 2018.

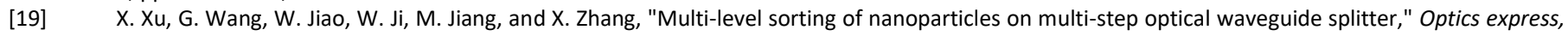
vol. 26, no. 22, pp. 29262-29271, 2018.

[20] S. Chen, R. Hao, Y. Zhang, and H. Yang, "Optofluidics in bio-imaging applications," Photonics Research, vol. 7, no. 5, pp. 532-542, 2019.

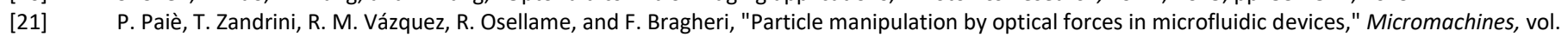
9, no. 5, p. 200, 2018.

[22] Z. Yao, C. C. Kwan, and A. W. O. W. Poon, "An optofluidic "tweeze-and-drag" cell stretcher in a microfluidic channel," Lab on a Chip, 2020.

[23] M. M. Wang et al., "Microfluidic sorting of mammalian cells by optical force switching," Nature Biotechnology, vol. 23, no. 1, pp. 83-87, 2005.

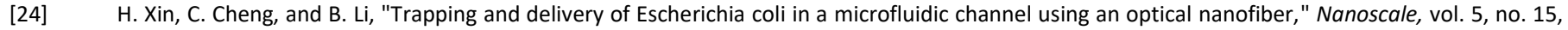
pp. 6720-6724, 2013.

[25] M. L. Povinelli et al., "Evanescent-wave bonding between optical waveguides," Optics Letters, vol. 30, no. 22, pp. $3042-3044,2005$.

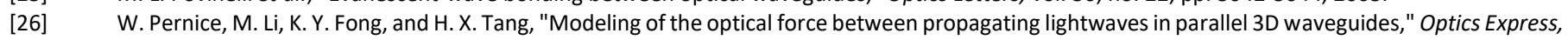
vol. 17, no. 18, pp. 16032-16037, 2009.

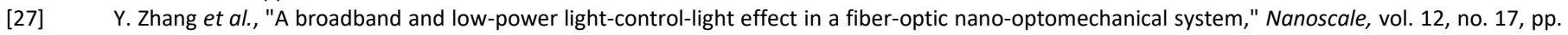
9800-9809, 2020.

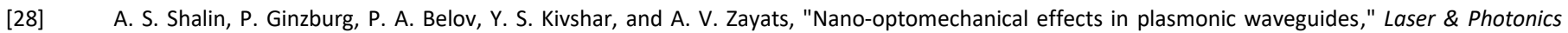
Reviews, vol. 8, no. 1, pp. 131-136, 2014. 
[29] L. Liu, J. Yue, and Z. Li, "All-optical switch based on a fiber-chip-fiber optomechanical system with ultrahigh extinction ratio," IEEE Photonics Journal, vol. 9, no. 3, pp. 1-8, 2017.

[30] P. Zhang, N.-H. Shen, T. Koschny, and C. M. Soukoulis, "Surface-plasmon-mediated gradient force enhancement and mechanical state transitions of graphene sheets," ACS Photonics, vol. 4, no. 1, pp. 181-187, 2017.

[31] A. Dash, S. Selvaraja, and A. Naik, "Probing optical mode hybridization in an integrated graphene nano-optomechanical system," JOSA B, vol. 37, no. 4, pp. 1122-1126, 2020.

[32] A. Dash, C. Samanta, P. Ranganath, S. Selvaraja, and A. Naik, "Optical gradient force for tuning, actuation, and manipulation of nonlinearity in graphene nanomechanical resonator," Journal of Optics, vol. 21, no. 6, p. 065803, 2019.

[33] B. Dong et al., "A silicon-nanowire memory driven by optical gradient force-induced bistability," Applied Physics Letters, vol. 107, no. 26, p. 261111, 2015.

[34] X. Yang, Y. Liu, R. F. Oulton, X. Yin, and X. Zhang, "Optical forces in hybrid plasmonic waveguides," Nano Letters, vol. 11, no. 2, pp. 321-328, 2011.

[35] H. Li, J. W. Noh, Y. Chen, and M. Li, "Enhanced optical forces in integrated hybrid plasmonic waveguides," Optics Express, vol. 21, no. 10, pp. 1183911851, 2013.

[36] A. T. Fanid and A. Rostami, "A proposal for wide range wavelength switching process using optical force," Physica Scripta, vol. 96, no. 12, p. 125537, 2021.

[37] X. Liu, C.-H. Yan, and J. A. Capobianco, "Photon upconversion nanomaterials," Chemical Society Reviews, vol. 44, no. 6, pp. 1299-1301, 2015.

[38] M. Haase and H. Schäfer, "Upconverting nanoparticles," Angewandte Chemie International Edition, vol. 50, no. 26, pp. 5808-5829, 2011.

[39] J. Yu et al., "Sensing and exploiting static Femto-Newton optical forces by a nanofiber with white-light interferometry," ACS Photonics, vol. 5, no. 8, pp. 3205-3213, 2018.

[40] H. Zheng et al., "Accurate measurement of nanomechanical motion in a fiber-taper nano-optomechanical system," Applied Physics Letters, vol. 115, no. 1, p. 013104, 2019.

[41] Y. H. Fu, A. I. Kuznetsov, A. E. Miroshnichenko, Y. F. Yu, and B. Luk'yanchuk, "Directional visible light scattering by silicon nanoparticles," Nature Communications, vol. 4, no. 1, pp. 1-6, 2013.

[42] W. Liu, J. Zhang, B. Lei, H. Ma, W. Xie, and H. Hu, "Ultra-directional forward scattering by individual core-shell nanoparticles," Optics Express, vol. 22, no. 13, pp. 16178-16187, 2014.

[43] F. Shen, N. An, Y. Tao, H. Zhou, Z. Jiang, and Z. Guo, "Anomalous forward scattering of gain-assisted dielectric shell-coated metallic core spherical particles," Nanophotonics, vol. 6, no. 5, p. 1063, 2017.

[44] C. F. Bohren and D. R. Huffman, Absorption and scattering of light by small particles. John Wiley \& Sons, 2008.

[45] E. D. Palik, Handbook of optical constants of solids. Academic Press, 1998.

[46] K. Luke, Y. Okawachi, M. R. Lamont, A. L. Gaeta, and M. Lipson, "Broadband mid-infrared frequency comb generation in a $\mathrm{Si}_{3} \mathrm{~N}_{4} \mathrm{microresonator,"}$ Optics Letters, vol. 40, no. 21, pp. 4823-4826, 2015.

[47] J. F. Shackelford and W. Alexander, CRC materials science and engineering handbook. CRC Press, 2000.

[48] Y. Xu et al., "Efficient polymer waveguide grating coupler with directionality enhancement," Optics Communications, vol. 463, p. 125418, 2020.

[49] M. Streshinsky et al., "A compact bi-wavelength polarization splitting grating coupler fabricated in a $220 \mathrm{~nm}$ SOI platform," Optics Express, vol. 21 , no. 25, pp. 31019-31028, 2013.

[50] C. Prokop, S. Schoenhardt, B. Laegel, S. Wolff, A. Mitchell, and C. Karnutsch, "air-suspended SU-8 polymer waveguide grating couplers," Journal of Lightwave Technology, vol. 34, no. 17, pp. 3966-3971, 2016. 